\title{
A Critical Analysis of Chromotherapy and Its Scientific Evolution
}

\author{
Samina T. Yousuf Azeemi and S. Mohsin Raza \\ Department of Physics, University of Balochistan, Quetta, Pakistan
}

\begin{abstract}
Chromotherapy is a method of treatment that uses the visible spectrum (colors) of electromagnetic radiation to cure diseases. It is a centuries-old concept used successfully over the years to cure various diseases. We have undertaken a critical analysis of chromotherapy and documented its scientific evolution to date. A few researchers have tried to discover the underlying scientific principles, but without quantitative study. Sufficient published material can be found about the subject that provides a complete system of treatment focused on the treatment methodologies and healing characteristics of colors. A number of studies have elaborated the relationship between the human body and colors. We also show the possibility of carrying out diverse research into chromotherapy that is pertinent to deciphering the quantum mechanical dipole moment of water molecules. The quantum mechanical dipole moment as a result of the absorption of different colors, we conjecture, produces charge quantization phenomena. This review illustrates that the development of science in the field of electromagnetic radiation/energy can be very helpful in discovering new dimensions of this old theory.
\end{abstract}

Keywords: charge quantization - chromotherapy - electromagnetic waves

\section{Hypothesis}

Newtonian ideas helped us to understand solid matter and moving objects found in the earth's gravitational field. Einstein, however, through his renowned equation $E=m c^{2}$, determined that energy and matter are dual expressions of the same universal substance (1).

The vibratory rate of a substance determines its density or its form as matter. A slowly vibrating substance is referred to as physical matter, whereas the subatomic (which vibrates at or above the speed of light) is subtle matter or pure light energy. Light is electromagnetic radiation, which is the fluctuation of electric and magnetic fields in nature. More simply, light is energy, and the phenomenon of color is a product of the interaction of energy and matter. The wavelength, frequency and quantity of energy of every colorful ray are fixed for each color; that is, a specific wavelength, a certain frequency and a particular amount of energy in that wave have been denominated as a distinct color. The human eye is sensitive to electromagnetic radiation only at wavelengths roughly between

For reprints and all correspondence: Samina T. Yousuf Azeemi, Department of Physics, Quetta, Pakistan. E-mail: saminatazayyen@yahoo.com
380 and $780 \mathrm{~nm}$. This small segment is called the visible spectrum or visible light. Visible light can be broken down into numerous electromagnetic frequencies, and frequency relates to a color of the rainbow: red, orange, yellow, green, blue, indigo, violet and all the vibrations thereof.

Newtonian concepts, limited to the observable, have also been applied by contemporary medicine, which is premised on the idea that the total picture becomes predictable by understanding and regulating the various material parts. That is, when a part of the body malfunctions, it is removed or replaced in a similar way to how we handle machinery. Alternatively, it is treated with chemical ingredients that often cause negative side effects. Simply stated, contemporary medicine examines the symptoms and influences or suppresses them, but it does not involve itself with a real source-diseased life energies. Einstein's approach deals with the human body not as an assemblage of chemical parts but as a total, complete system operating in harmony with the electromagnetic/energy system of the universe. The key to understanding chromotherapy/ vibrational healing lies not in the Newtonian mechanistic approach but in rerouting energy fields that form complex relationships with other fields such as those surrounding the physical/cellular substance and others relating to more nonphysical energies (1).

(c) The Author (2005). Published by Oxford University Press. All rights reserved.

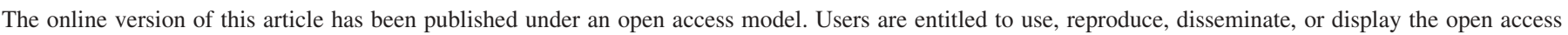

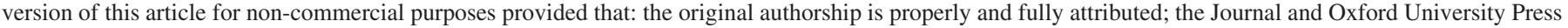

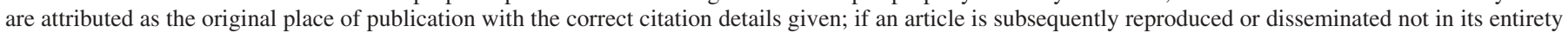
but only in part or as a derivative work this must be clearly indicated. For commercial re-use, please contact journals.permissions@oxfordjournals.org 
Every creature is engulfed in light that affects its health conditions (2). The human body, according to the doctrine of chromotherapy, is basically composed of colors. The body comes into existence from colors, the body is stimulated by colors and colors are responsible for the correct working of various systems that function in the body. All organs and limbs of the body have their own distinct color (3). All organs, cells and atoms exist as energy, and each form has its frequency or vibrational energy. Each of our organs and energy centers vibrates and harmonizes with the frequencies of these colors. When various parts of the body deviate from these expected normal vibrations, one can assume that the body is either diseased or at least not functioning properly. The vibratory rates inherent in the vibrational technique (chromotherapy) are such that they balance the diseased energy pattern found in the body. For in every organ there is an energetic level at which the organ functions best. Any departure from that vibratory rate results in pathology, whereas restoring the appropriate energy levels to the physical organs results in a healed body (1).

Chromotherapy is a narrow band in the cosmic electromagnetic energy spectrum, known to humankind as the visible color spectrum. It is composed of reds, greens, blues and their combined derivatives, producing the perceivable colors that fall between the ultraviolet and the infrared ranges of energy or vibrations. These visual colors with their unique wavelength and oscillations, when combined with a light source and selectively applied to impaired organs or life systems, provide the necessary healing energy required by the body. Light affects both the physical and etheric bodies. Colors generate electrical impulses and magnetic currents or fields of energy that are prime activators of the biochemical and hormonal processes in the human body, the stimulants or sedatives necessary to balance the entire system and its organs (Fig. 1).

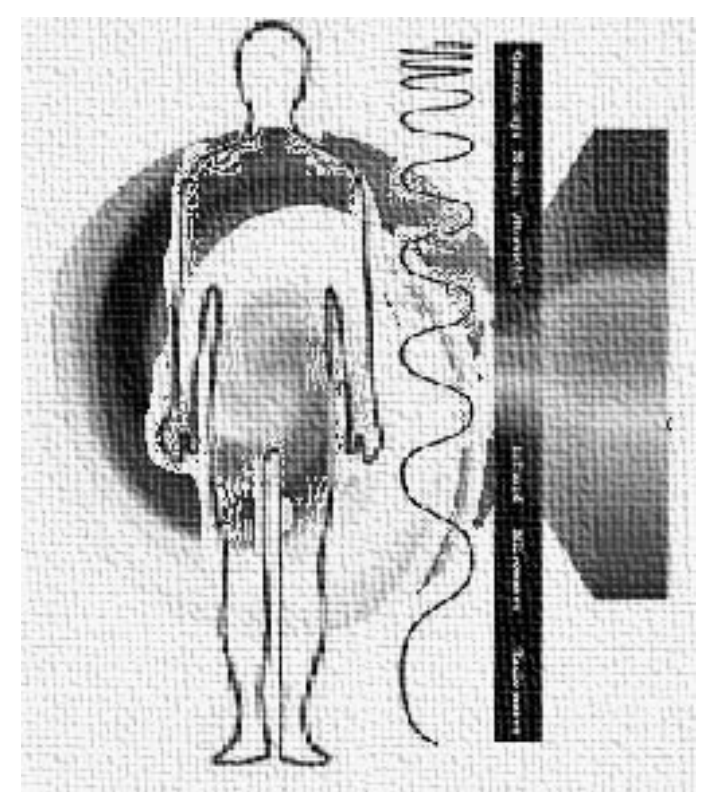

Figure 1. Human Body: conglomeration of colors.

\section{History of Chromotherapy}

\section{Physical View}

Ancient observation chromotherapy is a centuries-old concept. The history of color medicine is as old as that of any other medicine. Phototherapy (light therapy) was practiced in ancient Egypt, Greece, China and India. The Egyptians utilized sunlight as well as color for healing (4). Color has been investigated as medicine since $2000 \mathrm{BC}$ (5). People of that era were certainly unaware of the scientific facts of colors as medicine, but they certainly had faith in healing with colors. They used primary colors (i.e. red, blue and yellow) for healing as they were unaware of the mixing up of two colors. The science seems to have been silent at those times.

According to ancient Egyptian mythology, the art of chromotherapy was discovered by the god Thoth. In the hermetic traditions, the ancient Egyptians and Greeks used colored minerals, stones, crystals, salves and dyes as remedies and painted treatment sanctuaries in various shades of colors (6). The ancient Ayurvedic physician Charaka, who lived in the sixth century BC, recommended sunlight to treat a variety of diseases (7). In ancient Greece the physical nature of color was dominant. Color was intrinsic to healing, which involved restoring balance. Garments, oils, plasters, ointments and salves were used to treat disease. The Greeks were unaware of biological changes in the body as a result of color treatment; nevertheless, they had blind faith in the healing properties of colors. It is also interesting to know that they used both forms of treatment with colors: direct exposure to sunlight and indirect healing. In the indirect method, they used such materials as stones, dyes, ointments and plasters as the medium. What was missing in their medicinal use of color was water as a medium for the absorption of color, which later proved to be the best remedy for removing toxins from the body. This concept is common among all researchers working on hydrochromopathy (3).

Avicenna (AD 980) advanced the art of healing using colors. He made clear the vital importance of color in both diagnosis and treatment. According to Avicenna, 'Color is an observable symptom of disease.' He also developed a chart that related color to temperature and physical condition of the body. He used color treatment with the view that red moved the blood, blue or white cooled it and yellow reduced muscular pain and inflammation (6). Avicenna's work undoubtedly advanced the use of chromotherapy in those times. He discussed the properties of colors for healing and was the first to establish that the wrong color suggested for therapy would certainly elicit no response in specific diseases. For example, he observed that a person with a nosebleed should not gaze at things of a brilliant red color and should not be exposed to red light because this would stimulate the sanguineous humor, whereas blue would soothe it and reduce blood flow. This seems to be the practical understanding at the time, but we do not find discrete values of frequencies or energies associated with these colors. 
19th Century Ideas and Practices Pleasanton (1876) used only blue and stated that blue was the first remedy in case of injuries, burns or aches. He reported his findings on the effects of color in plants, animals and humans. He claimed that 'the quality yield and the size of grapes could significantly increase if they were grown in a greenhouse made with alternating blue and transparent panes of glass' (8). He also cured certain diseases and increased fertility as well as the rate of physical maturation in animals by exposing them to blue light. The same methodology employing the color blue was adopted by Hassan (1999), who found it to be very useful as a first-line treatment for injuries as well as for burns. Since, Pleasanton's work lacked scientific proof and evidence, no established rules were presented before the scientific societies, leading to a great gap between his work and the development of color/vibrational healing on scientific grounds. If work could be carried out even now on his great ideas, especially in agricultural development and in animals, researchers could make new discoveries.

Most areas that seem to have been ignored in the past were emphasized by Edwin Babbitt. Babbitt presented a comprehensive theory of healing with color. He identified the color red as a stimulant, notably of blood and to a lesser extent the nerves; yellow and orange as nerve stimulants; blue and violet as soothing to all systems and as having anti-inflammatory properties. Accordingly, Babbitt prescribed red for paralysis, physical exhaustion and chronic rheumatism; yellow as a laxative, emetic and purgative and for bronchial difficulties; blue for inflammatory conditions, sciatica, meningitis, nervous instability, headache, irritability and sunstroke. He also stated that 'all vital organs have direct connection with the skin through arteries, blood vessels and capillaries, and colour rays can affect the entire blood stream through circulation and elimination of toxins' (9). Babbitt also developed various devices, including a special cabinet called a thermolume, in which colored glass and natural light were used to produce colored light and a chrome disk - a funnel-shaped device fitted with a special color filter-was used to focalize light onto various parts of the body. He discussed in detail the effects of the reflection, absorption, transmission and polarization of light. Different patients were presented in his book who had been treated using color healing devices created by him. Babbitt also established the relationship between color and minerals, which he used as an addition to treatment with colored light, and he developed elixirs by irradiating water with sunlight filtered through colored lenses. He claimed that this 'potentized water' retained the energy of the vital element within the particular color filter used and had remarkable healing power (7).

Babbitt was in fact among the pioneers of modern chromotherapy. He used both direct and indirect methods of color treatment. He seemed to be well aware of the techniques and methodologies used in chromotherapy. His invention of different devices such as a special cabinet that used natural light to produce colored light by splitting it into seven colors, used for the focalization of light onto some particular area, worked quite effectively for healing wounds and stopping bleeding, headaches, etc. The actual energy to which he referred in potentized water was not calculated by any means. He did not explain the energy change in water, its quantum states and how different kinds of vibrations affect water in different manners. He did not explain about the potency of potentized water, but incredible for that time was is his correlation of magnetism with chromotherapy. His work on color healing, for the first time in history, proved to be comprehensive in taking both a physiological and a psychological approach. Any chromotherapist even nowadays can benefit from his work as he discussed appropriate colors for diseases in detail that in a way does not contradict to the facts newly established under the influence of science.

20th Century Scientific Emergence Ghadiali (1927) discovered the scientific principles that explain why and how different color rays have various therapeutic effects on the body. His Spectro-Chrome Encyclopaedia, is considered to be the first published book to explain the complete doctrine of chromotherapy. The rules explained in this book could be proved using any kind of modern techniques. Most chromopaths have used his technique $(1,10)$. He discovered that there is a unique color or energy vibration that either sedates or stimulates the stream of energy through a specific organ, causing a natural biochemical reaction. By knowing the action of different colors upon the different organs and systems of the body, one can apply the appropriate color that will balance the action of any organ or system that has become abnormal in its functioning or condition. When this balance is disturbed, mental and physical problems occur. The aim of the science of color healing is to cure disease by restoring normal balance of color energies of the body (11). Ghadiali established that particular areas of the body respond to particular colors; these areas are similar to what the ancients called 'chakras'. According to Klotsche, 'the chakras are areas of highly concentrated energy that are connected to various locations mainly along the spinal cord. These energy fields are related to the major organs in the body' (1). The concept of chakras is essentially an east Indian concept, which Ghadiali presented as the source of energies.

The work of Ghadiali actually demystified the theory of chromotherapy. Ghadiali's research stated: 'The colour bands of spectrograms are produced when a chemical element undergoes a process of combustion or vaporization that accelerates the motion of its atoms. The specific band of colours and dark lines emitted when a certain element is heated, are known as Fraunhauafer lines.' This procedure is commonly used to identify the chemical composition of a substance (with a photospectrometer) (11).

Contrary to accepted scientific theory, which assumes that each element is a unit, Ghadiali concluded that 'the chemical elements are colour compounds'. His results can be proved by any of the sophisticated equipment of modern science. 'A specific disease thus constitutes a specific imbalance of colour waves and by implication, chemical imbalance.' Ghadiali found that by treating the body with a particular color 
vibration, one could effectively reintroduce the appropriate biochemical elements into the body; he referred to this as color chemistry, certainly a new field of study. His results as published in the first decade of the twentieth century were advocated by Klotsche (1) in Colour Medicine: color medicine not only can heal the diseased frequency of the body but also can introduce actual chemical elements/vibration into the body in a non-toxic form.

During the nineteenth century the emphasis in science was exclusively on matter rather than on energy. As medicine came under the umbrella of science, it focused too much on the material physical body, ignoring the mind. With the advances in physical medicine and treatments such as surgery and antiseptic, interest in healing with colors declined.

Understanding Chakra and Color Ray Frequency In 1951 Takkata discovered that 'Colour Ray Frequency changes in atmosphere arising from the sunspots really affect the flocculation index of human blood albumin resulting in changes of menstrual cycles'. Takkata came up with experimental results on direct exposure to sunlight. He did not mention anything about material aids for providing a color deficient to the human body. Ott described Takkata's experiment in Part III of his series Colour and Light: Their Effect on Plants, Animals and People, published in 1987, and described how color rays from sunspots would alter a person's flocculation index. He further explained that there are different methods of applying colored light. It can be received through the skin or the eyes, which, in turn, has been found to stimulate the internal glands (10). Ott's work seems to be a continuation of Takkata's efforts; both have worked on the effects of light on blood, but Ott also described the different methods of chromotherapy. It is not clear in his work what parameters he has adopted to verify the effect of sunlight on skin. The same effect was also described in Babbitt's work, which is more informative and explanatory. It is very interesting that no chromopath has contradicted another's specific color treatment suggested for a specific disease. Ott also emphasized the biological functioning of the human body when chromotherapy is applied. He noted that different lights affect different enzymatic reactions for healing purposes (10). This was the first time that the effect of chromotherapy was tested at the DNA level.

As Ghadiadi, Klotsche correlated colors with chakras:

Red Root chakra

Orange Sacral chakra

Green Heart chakra

Blue Throat chakra

Indigo Brow chakra

Violet Crown chakra

White Perfect color blend

Each chakra energizes and sustains certain organs. The balance of the seven chakras activates healing by transmitting energy to the electromagnetic field around the body (1). 'The body has seven major energy centres known as chakras, each centres is responsive to a different colour. Chakra located at the sites of the major endocrine glands, corresponds to particular states of consciousness, personality types and endocrine secretions.

Approaches to Chromotherapy For new researchers, Klotsche discussed some useful points about chromotherapy as he practiced it, and he found it to be a complete therapeutic system for 123 major illnesses. He used single colors and also combinations of two or more colors for therapy and different techniques, namely, direct exposure and hydrochromopathy. He correlated the concepts of color healing with Einstein's mass-energy relationship, which seems quite accurate in terms of the concept of an energy field around the human body. His work seems to be an extension of Ghadiali's concept, but it proved to be more accurate. He emphasized the pros of chromotherapy - that it is safe, simple, economical and highly effective-but still his work lacks scientific proof on hydrochromopathy, which according to him was the best means of toxin elimination. We find no scientific calculations in his study; no spectroscopy has been conducted in this context.

Mester conducted experiments to determine the function of light in animal and human cells. The work of Mester resembles that of Azeemi and gives a clear picture of the effects of colors on the human body, whether applied directly to the skin or absorbed in such materials as water, oil and milk and then given to the patient. This could affect patients with hereditary diseases such as hypertension, thalassemia and diabetes. This work needs more research and a series of experiments should be carried out with certain biophysical applications. Azeemi discussed in detail the causes of diseases and suggested appropriate colors, which are very easy to understand and to use. He discussed in detail different methods of chromotherapy but emphasized hydrochromopathy. The complete methodology of chromotherapy as described by him is extremely useful and effective; undoubtedly, a new area of research has evolved with the publication of his book.

Hassan also adopted the methodology presented by Azeemi. His work is remarkable in the sense that he compiled all the concepts of chromopathy established so far. Chromotherapists of the past emphasized one thing and left others unattended. Some have emphasized direct exposure of the affected and diseased area to light. Some have talked about the materials, and others have emphasized only watching color. It is evident that all these methods focused only on the material aspect of chromotherapy, but Hassan produced a detailed study covering different aspects of chromotherapy, including the material aspect of healing as well as the electromagnetic transfer of color characteristics. Hassan (3) measured the production of a 32 su (sparkle units) charge in chromotized water due to the absorption of rays, but any theoretical explanation is missing. This was the first time in the history of chromotherapy that this kind of work had been done, but surprisingly Hassan did not proceed further to the spectroscopy of charged water. He also related seven musical tones to seven vibrational 
states and seven vitamins. He compared chromotherapy with all other therapeutic systems developed so far, with an in-depth explanation of the complete doctrine of chromotherapy (vibrational healing). He states: 'Every therapeutic system has its own doctrine or to say own point of view about the reasons and causes of diseases.' According to the theory of chromalux,

An electric charge is produced due to the influence of the vibrations of cosmic and colourful rays upon the brain cells. This electric charge takes the form of a current emitted where various cells collide with another. This collision results in formation of incalculable colourful vibrations, which can be termed as thought.

The cervical vertebrae is the main passage for the current that starts from the brain; if this bone fractures, the flow of current suffers a set back resulting in damage of the brain tissues (3).

He elaborated the technique of choosing the right color for specific diseases and explained the theory of the basic colors used for therapy and the combinations of different shades. Hassan's work stresses that a patient's history should be keenly observed before suggesting any color.

Color Psychology and Medicine For research techniques and impressive quantitative data, the world of color psychology and medicine is indebted to the recent efforts of Gerard (1970). He painstakingly reviewed the whole area of light, color and their psychophysiological influences. Probably for the first time, he tested the reactions of the entire organism, using advanced and modern techniques with colored light beamed onto the skin of the subject. Profiting from the experience of other scientists and the use of an electroencephalogram, he evolved new approaches and discovered a number of significant facts (12).

Physiologically, affective responses of subjects revealed that warm colors were useful in arousing those troubled with reactive depression or neurasthenia. They increased muscle tone or blood pressure in hypertensive individuals. Cool colors elicited the reverse affective responses in all of the same tests. Anxious subjects were actually calmed by these cooler colors, from the viewpoint of clinical psychology. This is an important finding in Gerard's work, as it reveals that cool colors can be effective as a tranquilizer in cases of tension and anxiety. Physiologically, all colors produced clinically tangible results. Exposure to warm colors increased respiratory movements, frequency of eye blinks, cortical activation and palmar conductance (arousal of the autonomic nervous system). Warm colors consistently showed a more pronounced pattern of stimulation. Cool colors showed opposite effects by acting as a relaxant and tranquilizer for anxious individuals, lowering blood pressure, providing relief from tension, alleviating of muscle spasms and reducing eye blink frequency. They also proved to be an aid for insomnia. Just as warm colors showed a consistently pronounced pattern of stimulation, cool colors showed a consistent pattern of relaxation. To summarize Gerard's research and testing, his scientific data showed that all colors affect all human both psychologically and physiologically in a specific manner.

\section{Metaphysical View}

Hassan related the human body to the electromagnetic energy glow surrounding every creature. In his view, this body or energy glow is responsible for keeping our body healthy. The same fact is described by Azeemi in his book Color Therapy thus: 'It is a wrong concept that our physical body is itself everything, but instead the electromagnetic glow (aura) around the body gives us the energy and transfers health or diseases to the physical body' (2).

These concepts received support from Russian scientists who worked in collaboration with an Indian researcher, Shah, using Kirlian technology. They took pictures of the electromagnetic energy glow around the human body and discovered that actual disease appears first in the aura and is then transferred to our physical body and can be detected 6-8 months prior to appearance in our physical body. Thus, they have established the fact that chromotherapy can be a preventive treatment (13). In support of this theory, Thelma Moss (14) noted: 'All seemingly solid objects in our world including our bodies are made up of the electromagnetic energy, the more dense the energy the more solid the object is.' This fact also demonstrates an idea of Einstein's quoted by Shah in his article 'Divine healing', that

if we are the objects with mass " $\mathrm{m}$ " and we expose ourselves to a very powerful and high intensity electromagnetic field, and then we will gradually be transformed from matter into energy. In the form of energy, if we obtain a superior level of consciousness then we will be able to direct the flow of energy and we will not be restricted by the limited dimensions of space-time. Therefore in this condition we can surpass the boundary of time to return back into the past or to travel in the future. In addition to the fact that we are an energy source that possesses conscious wisdom, we have the capability to transform ourselves back to the physical form (13).

After Einstein's statement, a new door could be opened to justify the electromagnetic body around the physical body, as posited by Klotsche in Colour Medicine and that only Einstein could demonstrate to the materialistic or mechanized Newtonbound world of the West through his mathematical energy formula $E=m c^{2}$. According to Einstein energy and matter are interchangeable and interconvertible. Klotsche explains this phenomenon thus:

We know that the vibratory rate of a substance determines its density or its forms as matter. When we recognize the vibratory patterns in the universe, 
i.e. the energy ranges or fields found on the cosmic electromagnetic scale, we will then be able to open the doors to the tremendous healing powers found in the subtle energy octaves of the cosmos. The visible light spectrum with its beneficial frequencies for the human body provides the preventing tool for healing. Colour Medicine is truly, the medicine of the future (1).

Chromotherapy provides colors to the electromagnetic body or the aura (energy field) around the body, which in turn transfers energy to the physical body. This makes chromotherapy the most effective among various therapies.

When we speak of color, we mean energy waves. Every color, each with its own frequency, is a form of energy (12). Ghadiali agreed that beaming a color or colors onto the skin acts as a form of feeding color to the body. Patterson of Stellar Research Corporation explains that 'light is the closest thing to pure energy that we can identify. Colour as pure vibrational energy is the rational therapy for maintaining health and overcoming disease' (15). When applied to the human body, light will provide all deficient energies since every color is associated with a quantity of energy. A concept from physics confirms the idea of chromotherapists that 'colors provide energies'. There have appeared no contradictions among any of the theories about chromotherapy presented, but still there are some areas of study which were not focused on in the past, including the study of the electromagnetic radiation glow around the human body and its quantization.

Colors have a profound effect on us at all levels - physical, mental and emotional. If our energy levels are blocked or depleted, then our body cannot function properly, and this in turn can lead to a variety of problems at different levels (16). This concept is also supported by Klotsche, who stated:

These interrelating systems of subtle forces recharge or rechannel energy into diseased areas where it is blocked or deficient, for disease is nothing more than a restriction of energy flow. As we know energy or vibrational flow along the path of least resistance and through the extra energy associate with the use of vibrational healing, the appropriate energies seek out the needed areas, freeing blocked energy where it is most required. The interaction between the dense physical energy of the body and the subtle energy, which controls many of the body functions or activities, is the key to understanding relationship between energy and matter (1).

This energy body can also be proved through photography, as described by Perry in scientific documentation of chromotherapy: in 1939, Kirlian discovered that if an object on a photographic plate is subjected to a high voltage electric field, an image is created on the plate. The image looks like a colored halo or a coronal discharge. This image is said to be the physical manifestation of the electromagnetic radiation around the body (aura), which allegedly surrounds every living thing (17).

Qalander (18) explained unrevealed facts about the human body and its energy glow in his work. This idea has also been used by Shah and Russian medical staff for treating patients. This discovery led to a new area of research; unfortunately, scientists even today have not yet explored the relationship between the basic science of electromagnetic energy around the body (aura) and chromotherapy. Orthodox medicine and science give their own explanations of how light works. These explanations are based on strictly physical functions and ignore the bioelectric energy field, which has been demonstrated or photographed with Kirlian devices (1). That electromagnetic energy can be moved through our auras into the physical body by light frequencies, using color medicine, can also be explained thus: 'All living things are moist; the moisture is transferred from the subject to the emulsion to the electric charge pattern on the films, causing a Kirlian image to appear' (14). This undoubtedly helps us understand how disease is cured according to the doctrine of chromopathy, but the area that should be emphasized is the quantum state of electromagnetic radiance around every living body.

\section{Applications of Chromotherapy}

Variation in Influences of Light During the 1950s, studies suggested that neonatal jaundice, a potentially fatal condition found in two-thirds of premature babies, could be successfully treated by exposure to sunlight. This was confirmed in the 1960s, and white light replaced high-risk blood transfusions in the treatment of this condition. Blue light was later found to be more effective and less hazardous than full-spectrum light (the most common form of treatment for neonatal jaundice). Comparison of blue light with turquoise for treatment of neonatal jaundice was carried out by Ebbesen (19). Bright white full-spectrum light is also now being used in the treatment of cancers, SAD (seasonal affective disorder, so-called winter depression), anorexia, bulimia nervosa, insomnia, jetlag, shift working, alcohol and drug dependency, and to reduce overall levels of medication. Schauss worked on the tranquilizing effect of colors and found that color reduces aggressive behavior and violence (20).

The blue light found to be successful in the treatment of neonatal jaundice has also been shown to be effective in the treatment of rheumatoid arthritis, as emphasized by Pleasanton in his work. In studies by McDonald, most of those exposed to blue light for variable periods of up to 15 min experienced a significant degree of pain relief. It was concluded that the pain reduction was directly related both to the blue light and to the length of exposure to it. Blue light is also used in healing injured tissue and preventing scar tissue, as well as for burns and lung conditions (2). In 1990, scientists reported to the annual conference of the American Association for the Advancement of Science on the successful use of blue light 
in the treatment of a wide variety of psychological problems, including addictions, eating disorders and depression. At the other end of the color spectrum, red light has been shown to be effective in the treatment of cancer and constipation and in healing wounds. As a result, color is becoming widely accepted as a therapeutic tool with various medical applications.

A new technique that has been developed over the past two decades as a result of pioneering research is photodynamic therapy (PDT). This is based on the discovery that certain intravenously injected photosensitive chemicals not only accumulate in cancer cells but also selectively identify these cells under ultraviolet light. These photosensitive chemicals then exclusively destroy the cancer cells when activated by red light, whose longer wavelength allows it to penetrate tissue more deeply than other colors. PDT can be used for both diagnosis and treatment. Thomas Dougherty, who developed PDT, reports that in a worldwide experiment more than 3000 people with a wide variety of malignant tumors have been successfully treated using this technique (21). Chromotherapy is now used to improve the performance of athletes; whereas red light appears to help athletes who need short, quick bursts of energy, blue light assists in performances requiring a steadier energy output.

By comparison, pink light has a tranquilizing and calming effect within minutes of exposure. It suppresses hostile, aggressive and anxious behavior. Pink holding cells are now widely used to reduce violent and aggressive behavior among prisoners, and some sources have reported a reduction of muscle strength in inmates within $2.7 \mathrm{~s}$. It appears that when in pink surroundings people can never become aggressive despite their desire, because the color saps their energy. In contrast, yellow should be avoided in such contexts because it is highly stimulating. Gimbel (22) suggested a possible relationship between violent street crime and sodium yellow street lighting.

Color, Brain and the Effects of Light Research in Russia during the 1960s showed that one in six experimental subjects could recognize color with their fingertips after only 20-30 min training, and blind people developed this sensitivity even more quickly. Understanding of these effects has come about only as a result of research into the hormones melatonin and serotonin, both of which are produced by the pineal gland in the brain. Melatonin is known to be the crucial chemical pathway by which animals respond to light and synchronize their bodily functioning with diurnal and seasonal variations. Serotonin is a very important neurotransmitter in the brain, whose action has been linked with mental disturbances such as schizophrenia and hallucinogenic states. Serotonin, a stimulant, is produced during daylight whereas the output of melatonin - which is linked with sleep - increases when it is dark and has a generally depressive effect. This is reversed when it is light and the production of melatonin drops. Its main site of action appears to be the hypothalamus, the part of the brain involved in mediating the effects of various hormones and in regulating emotions. However, changes in the output of melatonin in response to light influence every cell of the body, notably the reproductive processes, which are sensitive to such variations. High levels of melatonin have been found in women with ovulation problems and anorexia nervosa (a characteristic feature of which is amenorrhea, or absence of periods), in men with low sperm count and in people suffering from SAD, which usually occurs during winter. Research also confirmed that certain parts of the brain are not only light sensitive but actually respond differently to different wavelengths; it is now believed that different wavelengths (colors) of radiation interact differently with the endocrine system to stimulate or reduce hormone production (22).

This work has given a new dimension to chromotherapy: the use of colors in psychological disorders. SAD has become a very common problem nowadays, in England in particular, where the sun does not shine for up to 1 or 2 weeks, so that no light enters into the body. As a consequence, psychological diseases manifest, mainly in the form of depressions, which, according to chromopathy studies, are curable without any use of tranquilizers.

A detailed study of chromotherapy, with patients exposed to sunlight through color filters, was produced by Jacob. He adopted modern theories to prove the relationship between melatonin, light and color. Takkata was the first researcher to attempt to find a relationship between blood and sunlight. Jacob's work concerns hormonal changes as a result of exposure to sunlight. Of course, sunlight is a perfect blend of seven colors; different colors are responsible for the release of different kinds of hormones, which keeps us healthy. Jacob stated in his work that

Light is responsible for turning on the brain and the body. Light enters the body through the eyes and skin. When even a single photon of light enters the eye, it lights up the entire brain. This light triggers the hypothalamus, which regulates all life-sustaining bodily functions, the autonomic nervous system, endocrine system, and the pituitary (the body's master gland). The hypothalamus is also responsible for our body's biological clock. It also sends a message, by way of light, to the pineal organ, which is responsible for releasing one of our most important hormones, melatonin. The release of melatonin is directly related to light, darkness, colors, and the Earth's electromagnetic field. This necessary hormone affects every cell in the body. It turns on each cell's internal activities, allowing them to harmonize with each other and nature. The pineal gland is believed to be responsible for our feeling of oneness with the universe and sets the stage for the relationship between our inner being and the environment. If that relationship is harmonious, we are healthy, happy, and feel a sense of well-being. An imbalance in this relationship makes itself known in the form 
of disorders or disease in our physical, mental or emotional states. The Pineal is our "light meter", and receives information from the heavens above, to give us that sense of oneness with the universe, and from the Earth's electromagnetic field below to keep us grounded. A perfect balance is necessary to maintain our health and to keep us in harmony with the environment (22).

\section{Conclusions}

Many aspects of humankind's explorations are ignored, neglected or discarded. Color medicine is one of these neglected items. The common feature of every remedial and curative system of treatment, whether it is Ayurveda, allopathy, acupuncture, Unani, homeopathy, biochemic, magnetotherapy, physiotherapy, radiotherapy, aromatherapy, reflexology or chromotherapy, is to somehow apply vibrations of one kind or another in such a manner that the body can be put back on the health track. Most systems induce vibrations indirectly, but there are a few in which the vibrations are used directly upon the body, and chromotherapy is one of them.

Babbitt, Ghadiali and Azeemi revolutionized to the development of chromotherapy. Their ideas were carried out by other researchers. No controversies were found among the theories presented, in research work conducted in any area of the world.

We conjecture that color is a quantum state of matter. There are other quantum states such as charm, beauty, flavor, tenderness, etc. These quantum states are linked with each other via 'glucons' and form intermediatory energy fields known as 'quarks'. Quarks, if condensed, produce bosons, a fifth state of matter. The medium used in chromotherapy has never been explored in depth; for example, water, the main medium used in chromotherapy, has never been studied quantitatively in any of the research conducted in the context of chromotherapy. The literature exhibits a severe lack of scientific work pertaining to quantum physical states and optical mathematics. Similarly no proofs available on the basis of scientific calculations of chromotized water. We found no study of quantum states and the electromagnetic glow around the human body. Conductivity measurements of the chromotized water used in hydrochromopathy have not been emphasized by any researcher. Mass-energy-related measurements could be helpful in potentizing or chromotizing the liquid medium used for treatment. Relationships ought to be established between charged water, its energy states and its effect on the human body. The quantum mechanical dipole moment as a result of absorption of different colors, we conjecture, produces charge quantization phenomena. Chromotherapy as a system of treatment can benefit people because of its harmony with nature. Everything that exists in this world is a combination of different colors (2). In every creation of God, one color or another is dominant; as stated by Azeemi:

By using clay, a clay pot is repaired and piece of cloth mends a doll made of cloth, the plastic is used to repair the articles of plastic, then why light and colours cannot be used for the human health care which is the origin of man's creation. The holy scriptures say that existence of man is based upon various types of lights and colours. Then why a human being cannot be treated with colours (2).

Walker once said:

You realize you are part of the hologram of life, surrounded by an aura or energy field that radiates distinct colour and vibrations. The aura fingertips your soul and reflects your goodness, wellness, mental stability, maturity, emotional/inner turmoil or peaceful fulfilment. More of each of these qualities, peace, wellness, stability, maturity and fulfilment may become your ever present precious possession by the application of colour's power in our daily living (23).

\section{References}

1. Klotsche C. Colour Medicine. Arizona: Light Technology Publishing, 1993.

2. Azeemi, Khawaja Shamsuddin. Colour Therapy. Karachi: Al-Kitab Publications, 1999.

3. Hassan M. Chromopathy. Peshawar: Institute of Chromopathy, 2000.

4. Coclivo A. Coloured light therapy: overview of its history, theory, recent developments and clinical applications combined with acupuncture. Am J Acupunct 1999;27:71-83.

5. Anonymous. Colour history. Accessed at http://www. colourtherapyhealing.com/colour/colour_history.php.

6. Graham H. Discover Colour therapy. (2004) Ca USA: Ulysses Press 1998.

7. Anonymous. Light therapy. Accessed at http://www.holistic-online.com/ Light_Therapy/light_intro.htm (1998).

8. Pleasanton A. Blue and Sun Light. Philadelphia: Claxton, Reuser \& Haffelfinger, 1876.

9. Babbitt E. Principles of Light and Colour. MT, USA: Kessinger Publishing, 1942.

10. Ott J. Health and Light: The Effects of Natural and Artificial Light on Man and Other Living Things. Connecticut, USA: Devin-Adair Pub, 1972.

11. Ghadiali D. Spectrochrome Metery Encyclopedia. NJ, USA: Dinshah Health Society, 1997.

12. Perry R. Scientific documentation on colour therapy. Accessed at http:// www.rachelperry.net/SCIENTIFIC.html.

13. Shah J. Divine healing, Accessed at http://www.jmshah.com.

14. Moss T. Body Electric, New York, P. Tarcher, 1980.

15. Amber R. Colour Therapy. Sarta Fe, N.M., Aurora Press, 1964.

16. Birren F. Light, Colour and Environment. PA, USA: Schiffer Pub Ltd, 1988.

17. Kortkov K. Accessed at http://www.kirlianresearch.com.

18. Qalander B. LOH-O-QALAM, Maktaba Tajuddin Baba Auliya, Karachi, 1979.

19. Ebbesen F, Agati G, Pratesi R. Phototherapy with turquoise verses blue light. Archiv Des Childhood Fetal Neonatal Edn 2003;88:F430.

20. Schauss AG. Tranquilizing effect of colour reduces aggressive behaviour and potential violence. J Orthomol Psych 1979;4:218-21.

21. Gaham H. Then and now-Part 2. Accessed at http://www.innerself.com/ Health/Colour_Therapy_part_2.htm.

22. Peterson A. Our relationships with light and colour. Accessed at http:// www.inlightimes.com/2001/02/colour-light.htm (2001).

23. Walker M. Power of Colours. NY, USA: Avery Publishing Group, 1990. 


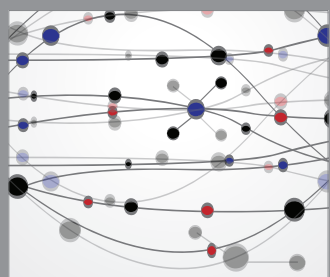

The Scientific World Journal
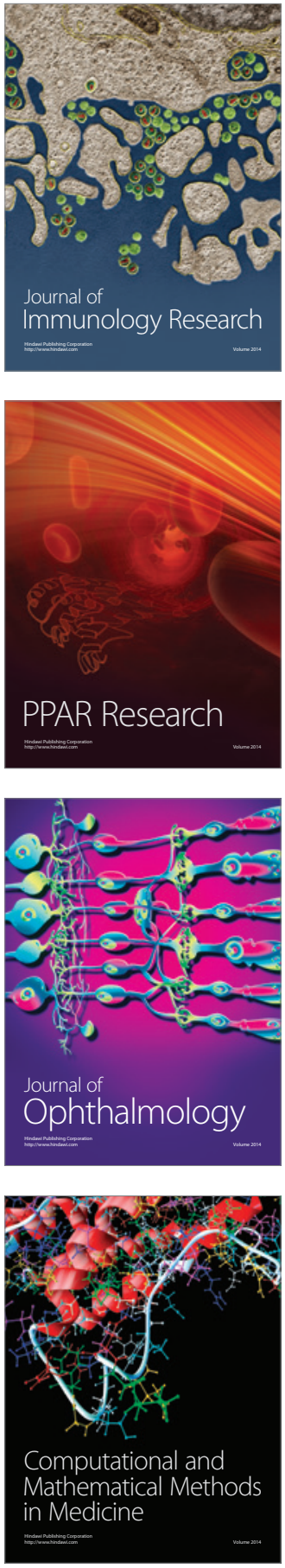

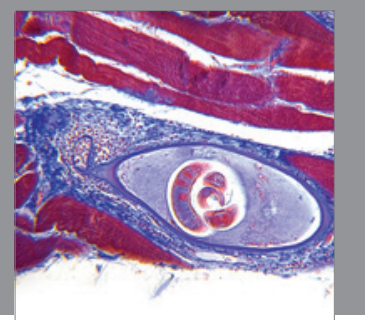

Gastroenterology

Research and Practice
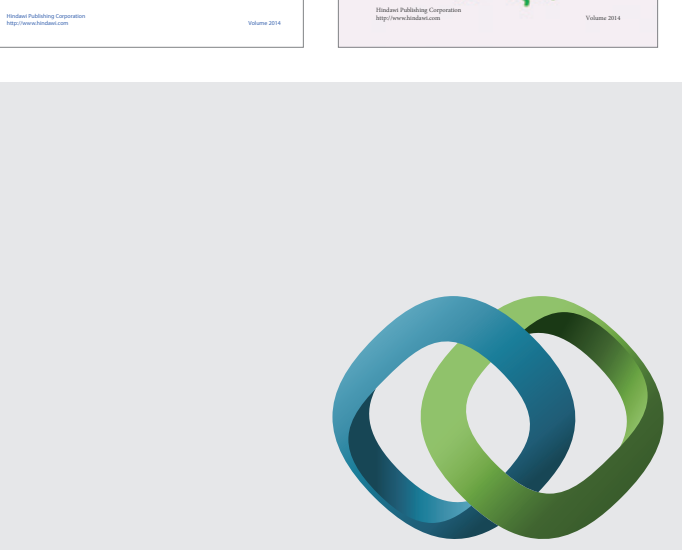

\section{Hindawi}

Submit your manuscripts at

http://www.hindawi.com
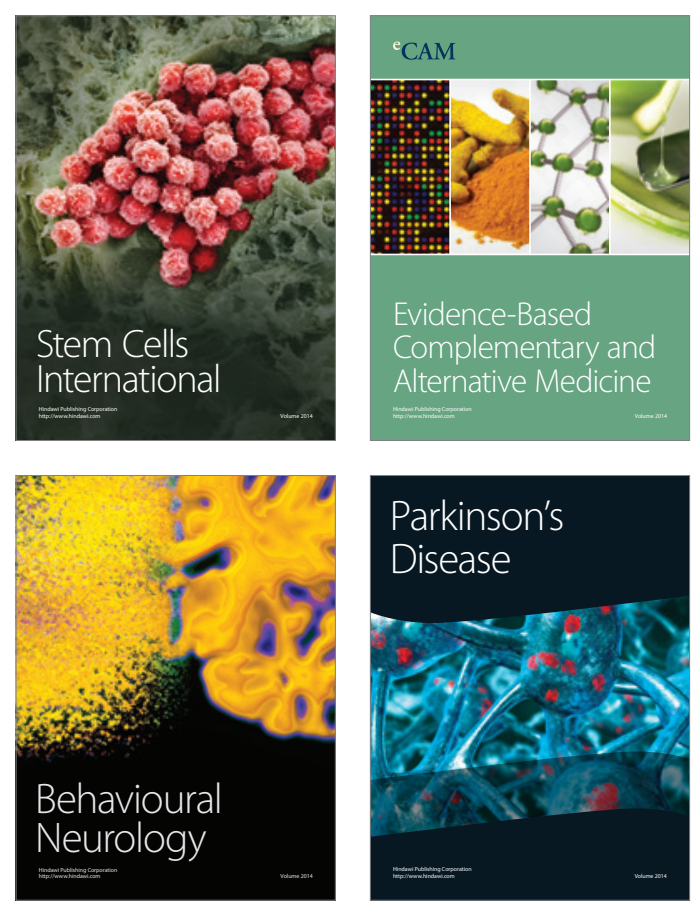

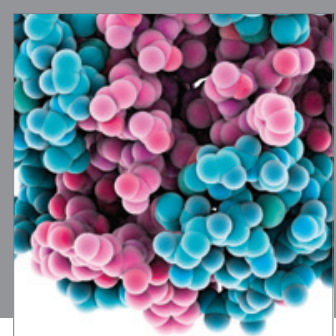

Journal of
Diabetes Research

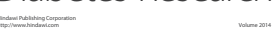

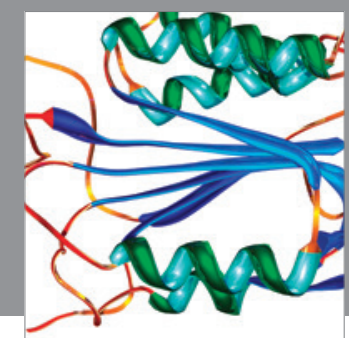

Disease Markers
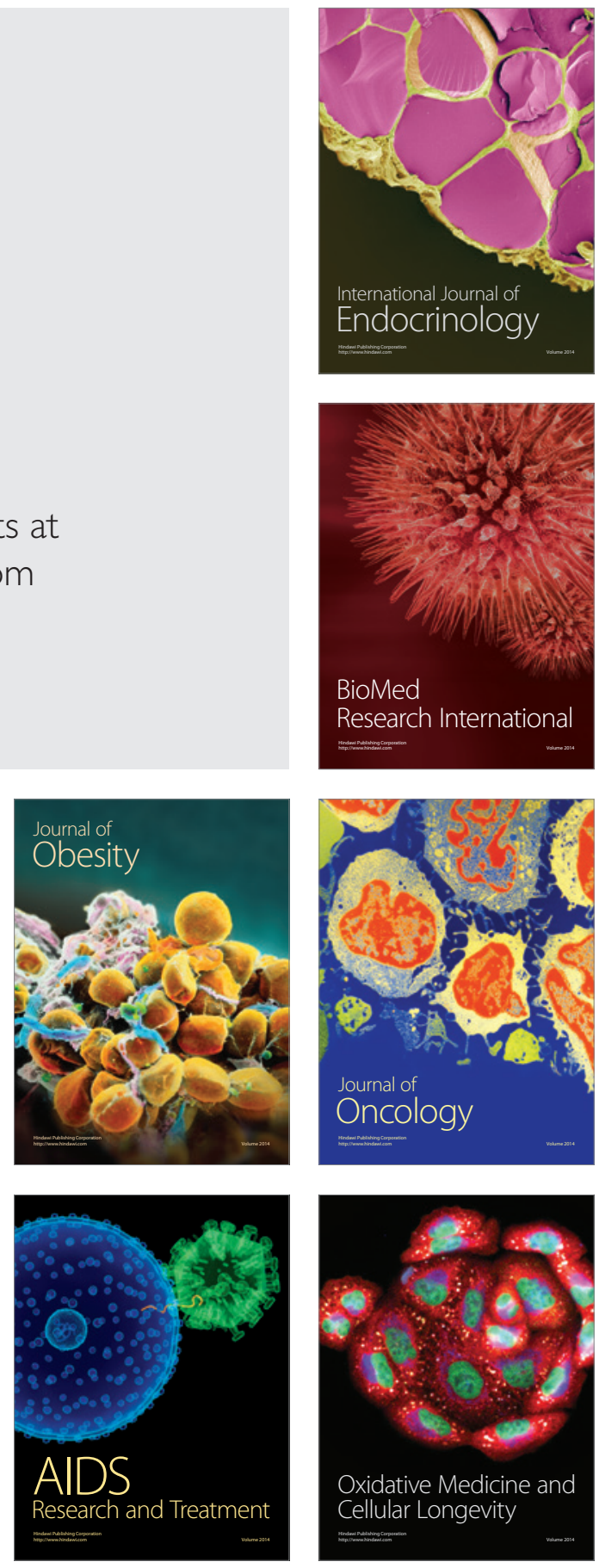